\title{
PERFIL CLÍNICO-LABORATORIAL E COMPROMETIMENTO VASCULAR EM PACIENTES COM DIABETES MELLITUS TIPO 2
}

Raquel Lima Chaves ${ }^{1}$

Mauren Isfer Anghebem-Oliveira ${ }^{2}$

CHAVES, R. L.; ANGHEBEM-OLIVEIRA, M. I. Perfil clínico-laboratorial e comprometimento vascular em pacientes com Diabetes Mellitus tipo 2. Arq. Cienc. Saúde UNIPAR, Umuarama, v. 20, n. 1, p, 59-65, jan./abr. 2016.

\begin{abstract}
RESUMO: O Diabetes mellitus (DM) é uma síndrome multifatorial cujas complicações matam 5\% da população mundial todo o ano. A hiperglicemia crônica característica do DM promove disfunção endotelial e o desenvolvimento de complicações microvasculares, como nefropatia, retinopatia e neuropatia, e macrovasculares como o acidente vascular cerebral e infarto agudo do miocárdio (IAM). O DM é uma patologia com altas taxas de morbidade e mortalidade, o que torna relevante a realização de estudos epidemiológicos para investigar suas características. O objetivo deste trabalho foi analisar o perfil clínico-laboratorial e a presença de complicações vasculares em pacientes com DM tipo 2 (DM2). Foram selecionados 300 pacientes com DM2 atendidos no Serviço de Endocrinologia e Metabologia do Hospital de Clínicas da Universidade Federal do Paraná (HC-UFPR). Dados clínico-laboratoriais foram coletados e analisados a partir do prontuário médico e do banco de dados do laboratório de análises clínicas do HC-UFPR. O projeto foi aprovado pelo Comitê de Ética em Pesquisa do Setor de Ciências da Saúde da UFPR (número CAAE: 01038112.0.0000.0102). Os participantes tinham média de idade de 62 anos, eram na maioria mulheres (73\%), apresentavam obesidade, hipertensão e dislipidemia. Concentrações de hemoglobina glicada $>7 \%$, indicando mal controle glicêmico, foram detectadas em 60,3\% dos pacientes com DM2 neste estudo. E 40,7\% dos participantes apresentaram ao menos uma complicação vascular. Os dados encontrados reforçam as estatísticas que pacientes com DM2 apresentam hipertensão, dislipidemias e obesidade, e estão em maior risco para complicações vasculares que podem causar morbidade e mortalidade.
\end{abstract}

PALAVRAS-CHAVE: Biomarcadores. Complicações vasculares. Diabetes mellitus tipo 2.

\section{CLINICAL AND LABORATORY PROFILE OF TYPE 2 DIABETES AND ITS VASCULAR COMPLICATIONS}

ABSTRACT: Diabetes mellitus (DM) is a multifactorial syndrome with complications that kill 5\% of the global population per year. Chronic hyperglycemia promotes endothelial dysfunction and the development of microvascular complications such as nephropathy, retinopathy and neuropathy, as well as macrovascular complications, such as stroke and acute myocardial infarction (AMI). DM is a disease with high morbidity and mortality, which makes it relevant to conduct epidemiological studies to investigate its features. The aim of this study was to evaluate clinical and laboratory profile and the presence of vascular complications in patients with type 2 DM (DM2). A total of 300 patients with DM2 treated at the Endocrinology and Metabolism Service at the Clinical Hospital of Federal University of Parana (HC-UFPR) were selected. Clinical and laboratory data were collected and analyzed from the medical records and laboratory database of HC-UFPR. This study was approved by the Research Ethics Committee of the UFPR Health Sciences Sector (CAAE number: 01038112.0.0000.0102). The mean age of participants was 62 years, with mostly women $(73 \%)$ presenting obesity, high blood pressure and dyslipidemia. Glycated hemoglobin concentrations $>7 \%$, indicating poor glycemic control, was detected in $60.3 \%$ of DM2 patients in this study, with $40.7 \%$ participants presenting at least one vascular complication. These results confirm the fact that DM2 patients have hypertension, dyslipidemia and obesity, and are at increased risk for vascular complications associated with high morbidity and mortality. KEYWORDS: Biomarkers. Type 2 diabetes. Vascular complications.

\section{Introdução}

Diabetes mellitus (DM) é uma síndrome metabólica caracterizada por hiperglicemia crônica que resulta de defeitos na secreção e/ou ação da insulina (ADA, 2015).

A classificação do DM preconizada pela Associação Americana de Diabetes, e amplamente utilizada pela comunidade científica, é baseada na etiologia das várias formas de diabetes, e as quatro categorias propostas são: DM tipo 1 (DM1); DM tipo 2 (DM2); Outros tipos específicos de DM; e DM gestacional ou DMG (SBD, 2015).

A maioria dos casos de DM (90\%) se enquadram no tipo 2, que é caracterizado pela resistência à insulina e/ou deficiência na sua ação ou secreção (ADA, 2015).

Múltiplas são as causas do DM2, mas sua etiologia ainda não é totalmente conhecida. Altas concentrações de glicose plasmática ativam vias metabólicas que favorecem o desenvolvimento e progressão de complicações micro e macrovasculares, como retinopatia, nefropatia diabética e doenças cardiovasculares (JAX, 2010).

Estima-se que 382 milhões de pessoas tenham diabetes no mundo e que dentro de vinte anos este número deva aumentar em 55\% (IDF, 2013). A prevalência do DM2 tem aumentado em decorrência dos hábitos de vida sedentários, mudanças na dieta, obesidade e envelhecimento populacional (ADA, 2014). Pelo fato do DM2 ser uma condição crônica de início silencioso, com sintomas nem sempre perceptíveis, quase metade dos indivíduos com DM estão sem diagnóstico, o que significa que não têm conhecimento de sua condição e dos riscos associados a ela (SACKS et al., 2011).

O DM está frequentemente associado à hipertensão, obesidade e dislipidemia. Alterações lipídicas em pacientes com DM2 são comuns e incluem aumento das concentrações séricas de triglicerídeos e LDL-colesterol, e redução de HDL-colesterol (LELLA; INDIRA, 2013). A aterosclerose, iniciada pela retenção de lipoproteínas aterogênicas no espaço subendotelial, é o principal fator de risco para doenças cardiovasculares. Além do risco associado à hiperglice-

DOI: https://doi.org/10.25110/arqsaude.v20i1.2016.5467

${ }^{1}$ Graduação em Farmácia; Escola de Saúde e Biociências; Pontifícia Universidade Católica do Paraná

${ }^{2}$ Professora Adjunta de Bioquímica Clínica; Escola de Saúde e Biociências; Pontifícia Universidade Católica do Paraná.

Autor correspondente: Profa. Dra. Mauren Isfer Anghebem-Oliveira. Pontifícia Universidade Católica do Paraná. Rua Imaculada Conceição, 155. Prado Velho - CEP 80215-901. Curitiba-PR. mauren_isfer@hotmail.com 
mia crônica, pacientes com DM2 e dislipidemia apresentam maior chance em desenvolver complicações cardiovasculares (HIMABINDU et al., 2013).

A avaliação do controle glicêmico de pacientes com DM2 é, tradicionalmente, feita por meio da dosagem laboratorial de glicemia e de hemoglobina glicada $\left(\mathrm{HbAl}_{\mathrm{C}}\right)$. A dosagem de glicemia reflete a concentração glicêmica do paciente no momento do teste; já os valores de $\mathrm{A} 1 \mathrm{C}$ refletem a glicemia média pregressa dos últimos dois a quatro meses (BHAKTHA et al., 2012).

Estudos multicêntricos de longa duração como o DCCT - Diabetes Control and Complication Trial (1986) e o UKPDS - United Kingdom Prospective Diabetes Study (1998), há mais de três décadas estabeleceram que o controle glicêmico adequado do paciente com DM promove uma significativa redução das complicações vasculares, melhorando a qualidade de vida dos afetados.

Aproximadamente 5\% de todas as mortes ocorridas anualmente no mundo são decorrentes das complicações do DM, sendo que as doenças cardiovasculares são responsáveis por mais de $50 \%$ da mortalidade entre os afetados (WHO, 2013). Levando-se em consideração a relevância do DM no âmbito da saúde pública há a necessidade de realizar estudos epidemiológicos para investigar suas características e estimular a implantação de medidas para redução de fatores de risco e melhora do controle glicêmico (UUSITUPA; TUOMILEHTO; PUSKA, 2011).

Com base nessas premissas, a proposta deste trabalho foi analisar o perfil clínico-laboratorial e a presença de complicações vasculares em pacientes com DM2. Estudos como o presente estimulam a promoção de políticas de saúde educativas e preventivas; além de facilitar a estratificação dos pacientes, a prevenção das complicações associadas às patologias e o direcionamento do tratamento clínico mais adequado.

\section{Métodos}

Trata-se de um estudo retrospectivo e transversal conduzidos com 300 pacientes com diagnóstico clínico e laboratorial prévio de Diabetes mellitus tipo 2 (DM2), atendidos na Unidade de Diabetes do Serviço de Endocrinologia e Metabologia do Hospital de Clínicas da Universidade Federal do Paraná (SEMPR-HC-UFPR).

\section{Seleção da amostra}

Os selecionados foram convidados a participar do estudo, e aderiram por meio da assinatura de Termo de Consentimento Livre e Esclarecido. O projeto teve a aprovação do Comitê de Ética em Pesquisa do Setor de Ciências da Saúde da UFPR (número CAAE: 01038112.0.0000.0102).

Foram excluídos do estudo indivíduos com idade inferior a 18 anos e aqueles diagnosticados com outros tipos de DM diferente do DM2.

\section{Coleta de dados}

A coleta de dados para este estudo foi realizada no HC-UFPR, mediante consulta ao prontuário médico de pacientes com DM atendidos no SEMPR, e consulta ao banco de dados do Laboratório de Análises Clínicas do HC-UFPR.

Dos prontuários médicos foram coletados os seguintes dados: idade, sexo, peso, altura, tipo de diabetes, uso de tabaco, presença de hipertensão arterial, presença de dislipidemia, uso de medicamentos e presença de complicações vasculares.

Do banco de dados laboratoriais foram coletados os seguintes resultados: glicemia em jejum, hemoglobina glicada $\left(\mathrm{HbA} 1_{\mathrm{C}}\right)$, perfil lipídico (colesterol total, HDL-Colesterol, LDL-Colesterol e triglicerídeos) e marcadores de função renal (uréia e creatinina).

O sobrepeso e a obesidade foram caracterizados pelo critério proposto pela Organização Mundial da Saúde (WHO, 2000).

Para definição de tabagismo, foi adotado o critério da Organização Mundial de Saúde, que considera fumante aquele que fumou mais de 100 cigarros na vida e fuma atualmente; ex-fumante quem fumou mais de 100 cigarros na vida e deixou de fumar há pelo menos um dia; e não fumantes são os que nunca fumaram, os que só experimentaram e os que fumaram menos de 100 cigarros na vida (WHO, 2009).

Os critérios adotados para a hipertensão foram os da V Diretrizes Brasileiras de Hipertensão Arterial da Sociedade Brasileira de Cardiologia (SBC, 2007) em que foi considerada hipertensão quando a pressão sistólica estava maior ou igual a $140 \mathrm{mmHg}$ e a diastólica maior ou igual a $90 \mathrm{mmHg}$, ou ainda quando o participante estava em uso de agentes anti-hipertensivos.

\section{Análise dos Dados}

Os dados clínicos e antropométricos retirados do prontuário médico e os resultados das análises laboratoriais dos pacientes foram armazenados em tabela própria do software de estatística Statistica 8.0 para as análises descritivas da amostra.

\section{Resultados e Discussão}

Foram analisados dados sobre o perfil clínico-laboratorial e a presença de complicações vasculares em pacientes com DM2, e a Tabela 1 resume as características antropométricas e demográficas dos participantes.

\section{Gênero, idade e etnia}

O DM2 pode ocorrer em qualquer idade, mas é geralmente diagnosticado após os 40 anos. O risco para DM2 aumenta com a idade, independente do gênero e da etnia (QIAO et al., 2003). A média de idade dos pacientes com DM2 avaliados neste estudo foi de aproximadamente 62 anos, compatível com a literatura. No entanto, é importante salientar que a prevalência de DM2 entre jovens e adolescentes tem crescido, sobretudo pelos hábitos alimentares e sedentarismo. Esses jovens que são diagnosticados com DM2 são, geralmente, de minorias étnicas como afro-americanos, mexicano-americanos e indígenas (PINHAS-HAMIEL; ZEITLER, 2007; SBD, 2015). 
Tabela 1: Caracterização antropométrica e demográfica da amostra.

\begin{tabular}{l|c}
\hline \multicolumn{1}{c|}{ Parâmetros } & DM2 (n=300) \\
\hline Gênero (Mulheres/ Homens) & $220 / 80$ \\
\hline Idade (anos) & $62,2 \pm 9,8$ \\
\hline IMC (kg/m2) & $31,0(26,7-34,7)$ \\
\hline Etnia (n, \%) & $192(64,0 \%)$ \\
Euro-brasileiros & $74(24,7 \%)$ \\
Afro-brasileiros & $34(11,3 \%)$ \\
Índios & $261(87 \%)$ \\
\hline Hipertensão arterial (n, \%) & $176(58,7 \%)$ \\
\hline Tabagismo (n, \%) & $16(5,3 \%)$ \\
Fumante & $108(36,0 \%)$ \\
Ex-fumante & $240(80 \%)$ \\
Não fumante & $163(54,3 \%)$ \\
\hline Dislipidemia (n, \%) & $259(86,3 \%)$ \\
\hline Uso de insulina (n, \%) & $240(80 \%)$ \\
\hline Uso de hipoglicemiantes orais (n, \%) & \\
\hline Uso de antilipemiante (n, \%) & $74(24,7 \%)$ \\
\hline Complicações do DM (n, \%) & $42(14,0 \%)$ \\
Retinopatia & $23(7,7 \%)$ \\
Infarto Agudo do Miocárdio & $21(7,0 \%)$ \\
\hline Nefropatia & \\
\hline Neuropatia & \\
\hline Nom & \\
\hline
\end{tabular}

$\mathrm{N}$, número de casos. Os resultados são apresentados como Média \pm 1 DP ou Mediana (intervalo interquartil, 25\%-75\%), para variáveis sem distribuição normal. IMC: Índice de massa corpórea. DAC: Doença arterial coronariana.

A etnia tem sido reportada em diversos estudos como um fator importante em relação a prevalência de DM, em que a prevalência é maior em indivíduos com descendência asiática, africana ou latina (PINHAS-HAMIEL; ZEITLER, 2007; NGUYEN et al., 2015). A população avaliada neste estudo é predominantemente euro-brasileira (64\%), e este resultado era esperado uma vez que a maioria dos participantes era de Curitiba e região metropolitana, reforçando os dados sobre a origem dessa população, em que cerca de 78\% é euro-descendente (IBGE, 2010).

\section{Índice de massa corpórea}

No presente estudo a obesidade, caracterizada pelo excesso de massa gorda, foi verificada pelo Índice de Massa Corpórea (IMC), seguindo o critério da Organização Mundial da Saúde, que enquadra um adulto em classes de peso, de acordo com o IMC obtido pelo peso (em $\mathrm{Kg}$ ) dividido pela altura ao quadrado (em metros; $\mathrm{m}^{2}$ ). O IMC entre $18,5 \mathrm{a}$ $24,99 \mathrm{~kg} / \mathrm{m}^{2}$ foi considerado normal (eutrófico); foi considerado sobrepeso quando os valores estavam entre 25 e 29,99 $\mathrm{kg} / \mathrm{m}^{2}$; obesidade classe I, quando estavam entre 30 e 34,99 $\mathrm{kg} / \mathrm{m}^{2}$; obesidade classe II, entre 35 e $39,99 \mathrm{~kg} / \mathrm{m}^{2}$; e obesidade classe III quando foram $\geq 40,00 \mathrm{~kg} / \mathrm{m}^{2}$ (WHO, 2000).

A obesidade é um dos principais fatores de risco para o DM2, e quanto maior o IMC, maior o risco em desenvolver a doença (KAHN; HULL; UTZSCHNEIDER, 2006;
SATTAR; GILL, 2014). A obesidade por si só favorece a resistência à insulina, pois o tecido adiposo libera substâncias, como ácidos graxos livres e citocinas inflamatórias, envolvidas no desenvolvimento da resistência à insulina e, consequentemente, do DM2 (BHATTARAI, 2009).

No presente estudo, a mediana para o IMC foi 31,0 $\mathrm{Kg} / \mathrm{m}^{2}$, compatível com a classe I de obesidade proposta pela Organização Mundial de Saúde (WHO, 2000). A maioria dos pacientes com DM2 apresentou sobrepeso ou obesidade, reforçando a ideia de que obesidade e resistência insulínica têm influência direta uma sobre a outra (BHATTARAI, 2009).

\section{Hipertensão arterial}

A hipertensão tem impacto significante no desenvolvimento de complicações vasculares, e, em conjunto com o DM, compartilham mecanismos comuns de lesão endotelial (LONG; DAGOGO-JACK, 2011).

Pacientes com DM tem maior risco para desenvolver hipertensão (SBC, 2007), fato confirmado no presente estudo, onde $87 \%$ dos participantes (261) foram classificados como hipertensos. A maioria dos pacientes com DM2 já se encontra hipertensa no momento do diagnóstico (SBD, 2015).

\section{Tabagismo}

O consumo de tabaco está associado ao risco aumentado para DM, e este risco aumenta proporcionalmente com a quantidade de cigarros fumados, porque a nicotina e o monóxido de carbono produzido durante a queima do cigarro agridem o endotélio vascular, causam inflamação e estresse oxidativo, os quais estão implicados com a resistência insulínica e, consequentemente, risco para desenvolvimento do DM (WILLI et al., 2007).

No presente estudo, quase $60 \%$ dos pacientes (180) com DM2 declararam ser fumantes, indicando a necessidade de campanhas de conscientização sobre os malefícios do cigarro, principalmente entre pacientes com DM, que já se enquadram em um grupo de risco para complicações vasculares.

\section{Dislipidemias}

Alterações no metabolismo dos lipídios são frequentes em pacientes com DM, e este dado foi reforçado no presente estudo, no qual $80 \%$ dos pacientes com DM2 apresentavam alguma dislipidemia e faziam uso de terapia antilipemiante. As doenças cardiovasculares são as que mais matam no mundo; e as alterações lipídicas são as principais responsáveis pela aterosclerose, base fisiológica da doença arterial coronariana (KONES; RUMANA, 2014). O DM é um fator de risco independente para doenças cardiovasculares, já que as vias envolvidas no metabolismo da glicose estão implicadas na disfunção endotelial, etapa crucial para o início da aterosclerose (PLUTZKY, 2011).

\section{Insulinoterapia e hipoglicemiante orais}

Neste estudo, 86,3\% dos participantes (259) faziam uso de hipoglicemiantes orais e 54,3\% em associação com 
insulina exógena, conforme reportado na Tabela 1. Devido ao caráter crônico do DM, a maioria dos pacientes não consegue manter um bom controle glicêmico apenas com dieta controlada e exercícios físicos, necessitando de terapia medicamentosa. O tratamento com hipoglicemiantes orais é a primeira escolha para pacientes com DM2, contudo, se estes fármacos não forem suficientes para controlar a hiperglicemia, é necessário o uso de insulina (HAAK, 2015). A alta frequência de pacientes DM2 em uso de insulina neste estudo indica um controle glicêmico inadequado.

\section{Complicações vasculares}

Dos 300 pacientes com DM2 avaliados neste estudo, $122(40,7 \%)$ apresentaram ao menos uma complicação vascular, e entre estes $28(9,4 \%)$ apresentou mais de uma complicação vascular. As frequências de complicações vasculares no grupo estudado estão demonstradas na Figura 1.

Figura 1: Distribuição das complicações vasculares entre os pacientes com DM2. Dos 300 pacientes com DM2 avaliados neste estudo, 122 apresentaram alguma complicação vascular, e 28 apresentaram mais de uma complicação. A associação mais comum foi a retinopatia e o IAM, infarto agudo do miocárdio.

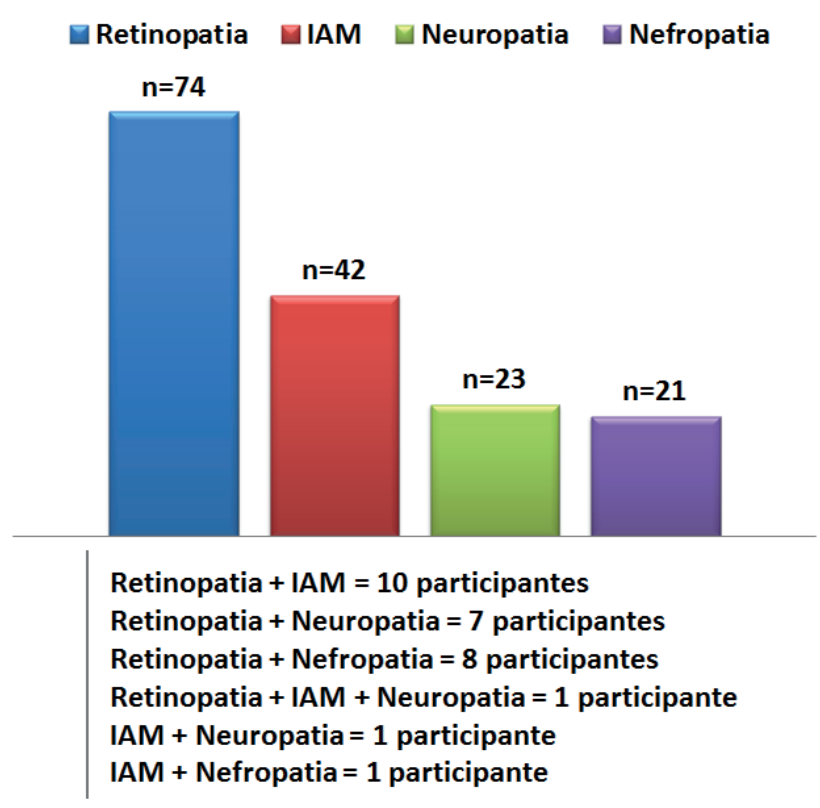

A hiperglicemia prolongada promove a glicação de proteínas, aumento da osmolaridade plasmática e aumento das concentrações de sorbitol dentro da célula, que acarreta mudanças na função dos nervos. O estresse oxidativo é um componente comum a todas as vias metabólicas envolvidas na disfunção endotelial (JAX, 2010). Processos pró-inflamatórios e pró-coagulantes, como o aumento na agregação plaquetária e disfunção na fibrinólise, além das alterações no metabolismo dos lipídeos e dislipidemias também contribuem para o risco aumentado para complicações vasculares no DM (BROWNLEE, 2001).

As complicações vasculares, sobretudo o IAM, são as principais causas de mortalidade entre os pacientes com DM (TRICHES et al., 2009). Neste estudo 122 participantes (40,7\%) apresentavam alguma complicação vascular, indi- cando que a hiperglicemia crônica e intensa favoreceu a disfunção endotelial e o aparecimento das complicações.

Santos Ade e colaboradores (2015) estudaram uma população de pacientes com DM2, com média de idade e distribuição de gêneros semelhantes à da população estudada aqui, e reportaram uma frequência de retinopatia superior a do presente estudo, $42,8 \%$ vs $24,7 \%$. Apesar dos autores não apresentarem as médias glicêmicas ou valores de HbA1c, é possível inferir que os pacientes abordados naquele estudo apresentavam um pior controle glicêmico comparado aos participantes do presente estudo, e quanto pior o controle glicêmico, maior o risco para complicações vasculares (BROWNLEE, 2005).

A retinopatia é a complicação vascular mais frequente entre pacientes com DM, e a principal causa de cegueira no mundo (HENRIQUES et al., 2015), e este dado foi corroborado pelo presente estudo (Tabela 1).

Também foram analisadas as concentrações dos parâmetros bioquímicos dos participantes, as quais estão relatadas na Tabela 2.

Tabela 2: Concentrações dos parâmetros bioquímicos.

\begin{tabular}{|c|c|c|}
\hline Parâmetros & DM2 $(n=300)$ & $\begin{array}{l}\text { Valores de } \\
\text { referência }\end{array}$ \\
\hline Glicemia (mg/dL) & $129,5(107,5-168,0)$ & $\leq 99,0 *$ \\
\hline $\operatorname{HbA1C}(\%)$ & $7,5(6,4-8,9)$ & $\leq 5,6 *$ \\
\hline $\begin{array}{l}\text { Colesterol total } \\
(\mathrm{mg} / \mathrm{dL})\end{array}$ & $175,8 \pm 39,9$ & $\leq 200 * *$ \\
\hline $\begin{array}{l}\text { HDL-Colesterol } \\
(\mathrm{mg} / \mathrm{dL})\end{array}$ & $41,3 \pm 10,1$ & $>60 * *$ \\
\hline $\begin{array}{l}\text { LDL-Colesterol } \\
(\mathrm{mg} / \mathrm{dL})\end{array}$ & $100,4 \pm 32,8$ & $<70 * *$ \\
\hline $\begin{array}{l}\text { Triglicérides (mg/ } \\
\mathrm{dL})\end{array}$ & $139,5(100-186)$ & $\leq 150 * *$ \\
\hline Ureia $(\mathrm{mg} / \mathrm{dL})$ & $35(29-44)$ & $15-44$ \\
\hline $\begin{array}{l}\text { Creatinina (mg/ } \\
\text { dL) }\end{array}$ & $1,65(0,75-1,00)$ & $0,6-1,2$ \\
\hline \multicolumn{3}{|c|}{$\begin{array}{l}\text { Os resultados são apresentados como Média } \pm 1 \text {-DP ou Mediana } \\
\text { (intervalo interquartil, } 25 \%-75 \% \text { ), para variáveis sem distribuição } \\
\text { normal. HbA1c: hemoglobina glicada. *Valores preconizados pela } \\
\text { Associação Americana de Diabetes (ADA, 2015). **Valores pre- } \\
\text { conizados pela V Diretriz Brasileira de Aterosclerose (XAVIER et } \\
\text { al., 2013). }\end{array}$} \\
\hline
\end{tabular}

\section{Marcadores do controle glicêmico}

Neste estudo, as concentrações de glicemia e HbA1c demonstram a consistência da classificação da amostra (pacientes com DM2) por estarem acima dos valores de referência (ADA, 2015). Neste caso, a utilidade clínica desses exames não seria para diagnóstico, mas sim para o monitoramento do paciente.

A avaliação do controle glicêmico de pacientes com DM é tradicionalmente feita por meio da dosagem laboratorial desses marcadores glicêmicos. A dosagem de glicemia reflete a concentração glicêmica do paciente no momento do teste; já os valores de $\mathrm{A} 1 \mathrm{C}$ refletem a glicemia média pregressa dos últimos dois a quatro meses, avaliando em longo prazo o controle glicêmico (SACKS et al., 2011). 
A variabilidade glicêmica é determinada pelos picos de hiperglicemia e hipoglicemia. A amplitude dessa variação é mais prejudicial que a própria hiperglicemia crônica, sendo um fator de risco independente para as complicações vasculares do DM. Por este motivo, o controle glicêmico dos pacientes com DM deve ser rigoroso (SMITH-PALMER et al., 2014).

As diretrizes nacionais e internacionais sobre diabetes recomendam como meta terapêutica concentrações de HbA1c menores que 7\% (SBD, 2015). Neste estudo, 181 participantes $(60,3 \%)$ apresentaram $\mathrm{HbA} 1 \mathrm{c} \geq 7 \%$. Este dado mostra o alto risco em que os pacientes se encontram.

A Figura 2 ilustra a distribuição dos participantes quanto a concentração de $\mathrm{HbA} 1 \mathrm{c}$, com base no valor proposto como meta terapêutica, $7 \%$.

Figura 2: Distribuição dos pacientes em relação as concentração de HbA1c. A curva em linha preta representa pacientes com HbA1c menor que 7,0\% (meta terapêutica ou bom controle glicêmico). A alta densidade mostra que os valores não foram dispersos. A curva em linha tracejada vermelha representa a distribuição dos pacientes com $\mathrm{HbA} 1 \mathrm{c}$ maior ou igual a $7 \%(60,3 \%)$, indicando mal controle glicêmico. A baixa densidade indica grande dispersão nos valores encontrados.

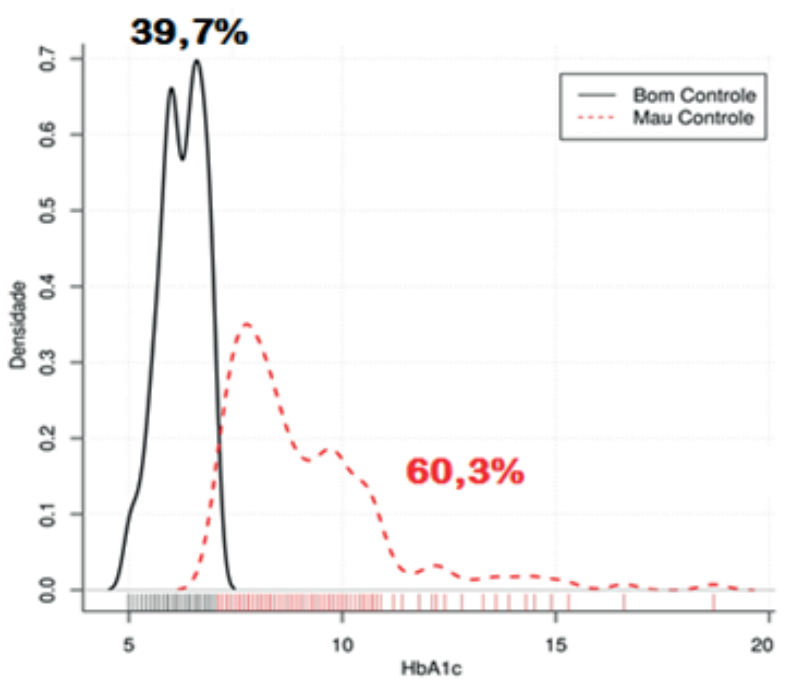

\section{Perfil lipídico}

O perfil lipídico compreende a quantificação sérica do colesterol total, HDL-c (HDL-colesterol), LDL-c (LDL-colesterol) e triglicérides. As concentrações de colesterol total e de triglicérides dos pacientes avaliados neste estudo estavam dentro do intervalo de referência proposto pela $\mathrm{V}$ Diretriz Brasileira de Dislipidemia (XAVIER et al., 2013), provavelmente pelo uso de antilipemiante $(80 \%)$. No entanto, as concentrações médias do HDL-c estavam abaixo da concentração associada à proteção cardiovascular (>60 mg/ $\mathrm{dL}$ ), e as concentrações de LDL-c, acima do recomendado $(<70 \mathrm{mg} / \mathrm{dL})$, indicando que não estão atingindo a meta terapêutica, mesmo com a terapia antilipêmica.

Pacientes com DM comumente apresentam alterações lipídicas caracterizadas por concentrações aumentadas de triglicérides, redução do HDL-c e aumento de LDL-c; que favorece o risco de desenvolver aterosclerose, e, consequentemente, o IAM (AL SIFRI et al., 2014; ADA, 2015a).

\section{Ureia e creatinina}

As concentrações de ureia e creatinina servem para avaliar a filtração glomerular, e monitorar o paciente com DM quanto ao desenvolvimento de nefropatia. A idade e a presença de diabetes favorecem a perda progressiva da função renal. Neste estudo, as concentrações de ureia estão dentro dos valores de referência. Já as concentrações médias de creatinina encontram-se aumentadas, sugerindo algum grau de lesão renal entre os participantes (Tabela 2). De fato, as concentrações mais elevadas de creatinina foram daqueles pacientes que apresentavam nefropatia (dados não mostrados).

\section{Conclusão}

Foram observadas características relevantes na amostra de pacientes com DM2 analisada neste estudo. A maioria dos participantes apresentava fatores de risco associados às complicações vasculares do diabetes, como tabagismo, obesidade e dislipidemia. Somado a isso, as concentrações de $\mathrm{HbA} 1 \mathrm{c}$ de $60,3 \%$ dos pacientes eram superiores à meta terapêutica de $7 \%$.

Conhecer as características clínico-laboratoriais dos pacientes com DM2 pode auxiliar na implementação de medidas que reduzam os fatores de risco associados à alta taxa de mortalidade entre estes pacientes.

A implantação de campanhas educativas e de ações preventivas pode reduzir os riscos das complicações associadas ao diabetes, melhorando a sobrevida dos afetados.

\section{Referências}

ADA. Diagnosis and classification of diabetes mellitus. Diabetes Care, v. 37 Suppl 1, p. S81-90. 2014.

Classification and diagnosis of diabetes. American Diabetes Association. Diabetes Care, v. 38, (Suppl. 1), p. 8, 2015.

Cardiovascular disease and risk management. Diabetes Care, v. 38, n.1, p. 9. 2015 a.

AL SIFRI, S. N. et al. Results of the Dyslipidemia International Study (DYSIS)-Middle East: clinical perspective on the prevalence and characteristics of lipid abnormalities in the setting of chronic statin treatment. Plos One, v. 9, n.1, p. 843-850, 2014.

BHAKTHA, G. et al. Is HbA1c a risk factor for type 2 diabetic subjects without macro and micro vascular complications? Arch Physiol Biochem. v. 118, n. 2, p. 6971, 2012.

BHATTARAI, M. D. Three patterns of rising type 2 diabetes prevalence in the world: need to widen the concept of prevention in individuals into control in the community. JNMA J Nepal Med Assoc. v. 48, n.174, p. 173-179, 2009. 
BROWNLEE, M. Biochemistry and molecular cell biology of diabetic complications. Nature, v. 414, n. 6865, p. 813$820,2001$.

BROWNLEE, M. The pathobiology of diabetic complications: a unifying mechanism. Diabetes, v. 54, n. 6 , p. 1615-1625, 2005.

DCCT - The Diabetes Control and Complications Trial: Design and methodologic considerations for the feasibility phase. The DCCT Research Group. Diabetes, v. 35, n. 5, p. 530-545, 1986.

HAAK, T. Combination of linagliptin and metformin for the treatment of patients with type 2 diabetes. Clin Med Insights Endocrinol Diabetes, v. 8, p. 1-6, 2015.

HENRIQUES, J. et al. Diabetic eye disease. Acta Med Port. v. 28, n.1, p. 107-113, 2015.

HIMABINDU, Y. et al. Correlations between anthropometry and lipid profile in type 2 diabetics. Indian J Endocrinol Metab. v. 17, n. 4, p. 727-729, 2013.

IBGE-Instituto Brasileiro de Geografia e Estatística. Censo demográfico 2010. Disponível em: $<$ http://www. ibge.gov.br/home/estatistica/populacao/condicaodevida/ indicadoresminimos/sinteseindicsociais2010/SIS_2010. pdf $>$. Acesso em: 10 maio 2013.

IDF - International Diabetes Federation. IDF Diabetes Atlas. Disponível em: < http://www.idf.org/sites/default/ files/EN_6E_Atlas_Full_0.pdf>. Acesso em: 20 set. 2013.

JAX, T. W. Metabolic memory: a vascular perspective.

Cardiovasc Diabetol. v. 9, p. 51, 2010.

KAHN, S. E.; HULL, R. L.; UTZSCHNEIDER, K. M. Mechanisms linking obesity to insulin resistance and type 2 diabetes. Nature, v. 444, n.7121, p. 840-846, 2006.

KONES, R.; RUMANA, U. Prevention of cardiovascular disease: updating the immensity of the challenge and the role of risk factors. Hosp Pract (1995). v. 42, n.1, p. 92$100,2014$.

LELLA, M.; INDIRA, K. A comparative study of efficacy of atorvastatin alone and its combination with fenofibrate on lipid profile in type 2 diabetes mellitus patients with hyperlipidemia. J Adv Pharm Technol Res. v. 4, n. 3, p. 166-170, 2013.

LONG, A. N.; DAGOGO-JACK, S. Comorbidities of diabetes and hypertension: mechanisms and approach to target organ protection. J Clin Hypertens (Greenwich), v. 13, n. 4, p. 244-251, 2011.

NGUYEN, T. H. et al. Type 2 diabetes among Asian Americans: Prevalence and prevention. World J Diabetes, v. 6, n. 4 , p. 543-547, 2015.
PINHAS-HAMIEL, O.; ZEITLER, P. Clinical presentation and treatment of type 2 diabetes in children. Pediatr Diabetes, v. 8, Suppl 9, p. 16-27, 2007.

PLUTZKY, J. Macrovascular effects and safety issues of therapies for type 2 diabetes. Am J Cardiol. v. 108, n. 3 Suppl, p. 25B-32B, 2011.

QIAO, Q. et al. Age- and sex-specific prevalence of diabetes and impaired glucose regulation in 11 Asian cohorts.

Diabetes Care, v. 26, n. 6, p. 1770-1780, 2003.

SACKS, D. B. et al. Guidelines and recommendations for laboratory analysis in the diagnosis and management of diabetes mellitus. Clin Chem. v. 57, n. 6, p. e1-e47, 2011.

SANTOS, ADE L. et al. Microvascular complications in type 2 diabetes and associated factors: a telephone survey of self-reported morbidity. Cien Saude Colet. v. 20, n. 3, p. 761-770, 2015.

SATTAR, N.; GILL, J. M. Type 2 diabetes as a disease of ectopic fat? BMC Med. v. 12, p. 123, 2014.

SBC - Sociedade Brasileira de Cardiologia. v diretriz brasileira em hipertensão arterial. arq Bras Cardiol. v. 89, n. 3, p. 24-79, 2007.

SBD - Diretrizes da Sociedade Brasileira de Diabetes: 20142015. Disponível em: <http://www.diabetes.org.br/images/ pdf/diretrizes-sbd.pdf $>$. Acesso em: 12 jan. 2015.

SMITH-PALMER, J. et al. Assessment of the association between glycemic variability and diabetes-related complications in type 1 and type 2 diabetes. Diabetes Res Clin Pract. v. 105, n. 3, p. 273-284, 2014.

TRICHES, C. et al. [Macrovascular diabetic complications: clinical characteristics, diagnosis and management]. Arq Bras Endocrinol Metabol. v. 53, n. 6, p. 698-708, 2009.

UKPDS. Intensive blood-glucose control with sulphonylureas or insulin compared with conventional treatment and risk of complications in patients with type 2 diabetes (UKPDS 33). UK Prospective Diabetes Study (UKPDS) Group. Lancet, v. 352, n. 9131, p. 837-853, 1998.

UUSITUPA, M.; TUOMILEHTO, J.; PUSKA, P. Are we really active in the prevention of obesity and type 2 diabetes at the community level? Nutr Metab Cardiovasc Dis. v. 21, n. 5, p. 380-399, 2011.

WHO. Obesity: Preventing and managing the global epidemic. Report of a WHO Consultation. Geneva: World Health Organization, 2000.

Guidelines for the prevention, management and care of diabetes mellitus. Geneva World Health Organization, p. 1-82, 2009.

The top 10 cause of death. Disponível em: <http:// 
www.who.int/mediacentre/factsheets/fs310/en/index.html>. Acesso em: 20 out. 2013.

WILLI, C. et al. Active smoking and the risk of type 2 diabetes: a systematic review and meta-analysis. JAMA, v. 298, n. 22, p. 2654-2664, 2007.

XAVIER, H. T. et al. V Diretriz brasileira de dislipidemias e prevenção da aterosclerose. Arq Bras Cardiol. v. 101 Suppl 1, p. 1-22, 2013.

Recebido: $10 / 12 / 2015$

Aceito: $20 / 04 / 2016$ 